\title{
Perfil epidemiológico de pacientes acometidos por acidente vascular encefálico cadastrados na Estratégia de Saúde da Família em Diamantina, MG Epidemiological profile of stroke survivors registered at the Health Family Strategy of Diamantina, $M G$
}

\author{
Hércules Ribeiro Leite, Ana Paula Nogueira Nunes ${ }^{2}$, Clynton Lourenço Corrêa ${ }^{3}$
}

Estudo desenvolvido no Depto. de Fisioterapia da UFVJM Universidade Federal dos Vales do Jequitinhonha e Mucuri, Diamantina, MG, Brasil

1 Fisioterapeuta; mestrando em Fisiologia e Farmacologia na UFMG

2 Fisioterapeuta; mestranda em Epidemiologia na UFMG

3 Fisioterapeuta; Prof. Dr. adjunto da Universidade Federal do Paraná - Litoral, Matinhos, PR

ENDEREÇO PARA

CORRESPONDÊNCIA

Hércules R. Leite

R. Otacílio Negrão de Lima

181 Centro

35774-000 Paraopeba MG

e-mail:

herculesfisio@hotmail.com; clynton@ufpr.br

APRESENTAÇÃo

maio 2008

ACEITO PARA PUBLICAÇÃO mar. 2009
Resumo: O acidente vascular encefálico (AVE) pode reduzir a capacidade de realizar tarefas funcionais, limitar a independência e a qualidade de vida dos indivíduos. O trabalho apresentou dois objetivos: identificar o perfil epidemiológico dos sujeitos com hemiplegia no município de Diamantina, MG, e implementar uma ação conjunta entre a universidade e as autoridades locais para atendê-los. Dos 82 pacientes cadastrados nas unidades de Estratégia de Saúde da Família (ESF), 51 foram contatados em seus domicílios e entrevistados usando roteiro semiestruturado. A média de idade foi 67,8 e o tempo de AVE, de 6,7 anos; $50 \%$ eram analfabetos e $41,2 \%$ mantinham-se com 1 salário mínimo por mês. Dentre os fatores de risco anteriores ao episódio de $\mathrm{AVE}$, foram relatados dieta inadequada, inatividade física, tabagismo, etilismo e histórico de AVE paterno e/ou materno. $\mathrm{Na}$ época da pesquisa, 78,4\% eram hipertensos; e 35,3\% nunca tinham feito fisioterapia após o AVE. Assim, são propostas: inserção de fisioterapeutas nas unidades ESF, mudanças no modo de vida dos indivíduos, bem como melhorias ou modificações nas estratégias de políticas de saúde na região estudada.

Descritores: Acidente cerebral vascular/epidemiologia; Fisioterapia (Especialidade)

ABSTRACT:Stroke can bring limitations to functional task performance, thus reducing patients' independence and quality of life. The purpose of this study was to draw the epidemiological profile of hemiparetic, stroke survivors in the city of Diamantina, MG, and to foster a joint program of action by the university and local public health service, in order to attend to these subjects. From 82 subjects registered at the Health Family Strategy units (HFS), 51 were contacted and interviewed, by using a semi-structured questionnaire. Their mean age was 67.8 years old, and time since onset of stroke, 6.7 years; $50 \%$ were illiterate and $41.2 \%$ lived on one monthly minimum wage. Reported risk factors prior to the stroke were unhealthy diet, physical inactivity, alcoholism, smoking, and family history of stroke (father or mother). At the time of the study $78.73 \%$ had high blood pressure and $35.29 \%$ had never undergone physiotherapy treatment after the stroke episode. Hence, inclusion of physical therapists in HFS, changes in subjects' lifestyle, and improvement or changes in local health policies are imperative.

KeY words: Physical therapy (Specialty); Stroke/epidemiology 


\section{INTRODUÇÃO}

O acidente vascular encefálico (AVE) é caracterizado por um distúrbio neurológico focal, ou às vezes global, durando mais que 24 horas, com desenvolvimento rápido dos sintomas ${ }^{1}$. Podem ser classificados como isquêmicos ou hemorrágicos. Os isquêmicos ocorrem por obstrução das principais artérias que levam sangue ao encéfalo, onde as áreas por elas irrigadas deixam de receber sangue oxigenado. Os hemorrágicos ocorrem por ruptura de uma dessas artérias do encéfalo, levando ao sangramento intraencefálico. O AVE isquêmico é o mais comum, ocorrendo em aproximadamente $88 \%$ dos casos, porém sua mortalidade é menor, quando comparado ao de caráter hemorrágico, em 15 a $20 \%$ dos casos 2 .

Os défices apresentados após o acidente incluem deficiência nas funções motoras, sensitivas, mentais, perceptivas e da linguagem, dependendo da localização da artéria acometida, da extensão da lesão e da disponibilidade de fluxo colateral3. Os sintomas neurológicos podem refletir a localização e o tamanho do AVE, porém não os diferem claramente quanto ao tipo de acidente vascular ${ }^{2}$.

Dentre 35 milhões de mortes atribuídas às doenças crônicas que ocorreram em todo o mundo em 2005, o AVE foi responsável por 5,7 milhões $(16,6 \%$ ) das mortes, sendo que $87 \%$ ocorreram em países subdesenvolvidos. Dessa forma, o AVE é um problema de saúde mundial ${ }^{4}$. Nos Estados Unidos, a incidência é de 700.000 casos por ano ( 165.000 correspondem a óbitos) $)^{5}$, e os respectivos custos anuais chegam a aproximadamente 58 bilhões de dólares6. Na América Latina, o AVE é causa permanente de morbidade $^{4,5}$ e mortalidade entre adultos ${ }^{7}$. Estudo realizado na América do Sul revela uma incidência de 35 a 183 casos por 100.000 habitantes. No Brasil, as taxas de mortalidade ajustadas à idade para o AVE estão entre as maiores em nove países da América Latina ${ }^{8}$.

Segundo o Datasus9 ${ }^{9}$, órgão vinculado ao governo federal, a taxa de mortalidade em Minas Gerais e em Diamantina em 2003 foi respectivamente de 51 e 45,2 por 100.000 habitantes, na po- pulação acima de 40 anos. Segundo Bueno et al. ${ }^{10}$, que analisaram os coeficientes de mortalidade por doenças cerebrovasculares no município de Diamantina de 1998 a 2006, foram encontradas taxas superiores desses óbitos em comparação com o estado de Minas Gerais em todos os anos analisados.

Este estudo teve por objetivo identificar o perfil epidemiológico dos pacientes com hemiplegia no município de Diamantina, MG, e implementar ações conjuntas entre a universidade e as autoridades locais da região estudada, para criar políticas locais de saúde de atendimento a esses pacientes.

\section{METODOLOGIA}

Pela revisão do cadastro de 82 pacientes com diagnóstico clínico de AVE nas áreas de abrangência das unidades do programa Estratégias de Saúde da Família (ESF) do município de Diamantina, os indivíduos foram contatados em seus domicílios e entrevistados pessoalmente por um único entrevistador treinado. Os pacientes que apresentaram outras doenças neurológicas e/ou ortopédicas associadas foram excluídos do estudo. Assim, 51 participantes do estudo assinaram o termo de consentimento livre e esclarecido. Este projeto foi aprovado pelo Comitê de Ética em Pesquisa da UFVJM - Universidade Federal dos Vales do Jequitinhonha e Mucuri. Para a coleta de dados, os coordenadores de todas as unidades ESF de Diamantina autorizaram a realização da pesquisa, após esclarecimentos sobre os objetivos desta pesquisa.

Inicialmente os pacientes foram submetidos ao miniexame do estado mentall $^{11}$, um teste que avalia o estado mental geral considerando orientação têmporo-espacial, memória recente, cálculo, linguagem e praxia motora, com pontuação de 0 a 30. O escore preditivo de normalidade varia de acordo com o grau de escolaridade do sujeito: de 18 (para analfabetos), 21 (para indivíduos com 1 a 3 anos de escolaridade), 24 (para indivíduos com 4 a 7 anos de escolaridade), a 26 (para aqueles com mais de 7 anos de escolaridade) ${ }^{12}$. Os pacientes que obtiveram valores abaixo do determinado não poderiam responder às perguntas do roteiro, sendo estas então feitas a seus respectivos cuidadores. As perguntas do roteiro semi-estruturado envolviam: nome, sexo, idade, grau de instrução, renda familiar, tempo de tratamento na fisioterapia, tempo decorrido desde o primeiro AVE, dimídio acometido, deambulação, tipo de marcha, uso de órteses, alterações na fala, hipertensão arterial sistêmica (HAS), hábitos de vida anteriores ao episódio de AVE (tabagismo, alcoolismo, dieta alimentar e atividade física) e histórico de AVE paterno e/ou materno.

A análise estatística dos dados foi descritiva simples, onde as variáveis qualitativas foram apresentadas em freqüências relativas (percentuais) e freqüências absolutas (N), e as variáveis quantitativonuméricas, em média e desvio-padrão.

\section{RESULTADOS}

Dos 82 pacientes cadastrados nas unidades ESF, 3 não contemplaram os critérios de inclusão do estudo e 28 não foram encontrados em seus domicílios. Assim, a amostra foi de 51 indivíduos,

Tabela 1 Características sociodemográficas e do AVE dos sujeitos da amostra $(\mathrm{n}=51)$

\begin{tabular}{lcc}
\hline Característica & $\mathrm{n}$ & $\%$ \\
\hline Idade (anos, $\mathrm{m} \pm \mathrm{dp})$ & \multicolumn{2}{c}{$67,8 \pm 13,6$} \\
Sexo masculino & 25 & 49 \\
$\quad$ feminino & 26 & 51 \\
Renda familiar & & \\
1 salário & 21 & 41 \\
2 salários & 10 & 20 \\
3 salários & 10 & 20 \\
4 salários ou + & 8 & 16 \\
0 salário & 2 & 4 \\
Escolaridade & & \\
Analfabeto & 25 & 49 \\
Fundamental completo & 2 & 4 \\
Fundamental incompleto & 22 & 43 \\
Médio completo & 1 & 2 \\
Superior & 1 & 2 \\
\hline Tempo de AVE & $6,7 \pm 5,6$ \\
$\quad$ (anos, m \pm dp) & 25 & 49 \\
Dimídio esquerdo & 22 & 43 \\
$\quad$ direito & 4 & 8 \\
$\quad$ ambos & & \\
\hline
\end{tabular}

$\mathrm{m} \pm \mathrm{dp}=$ média \pm desvio padrão 


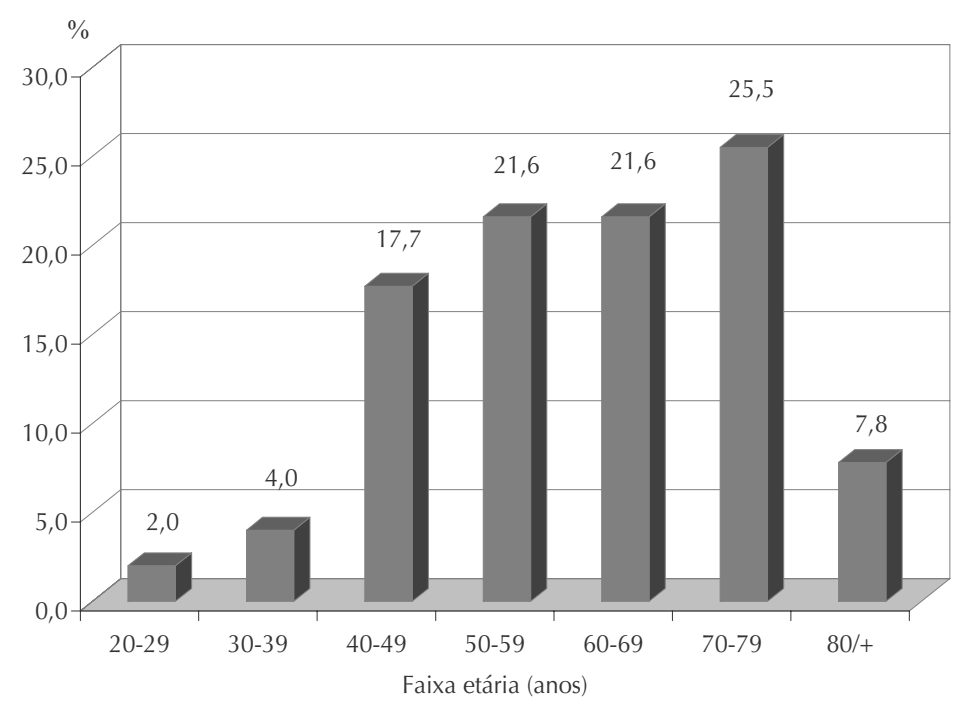

Gráfico 1 Distribuição (\%) da idade em que ocorreu o primeiro episódio de acidente vascular encefálico por grupo etário dos sujeitos $(n=51)$, segundo informado por eles terapêutica (necessidade constante do cuidador ou do terapeuta para que o paciente deambule em curta distância).

\section{DISCUSSÃO}

Segundo o Ministério da Saúde, a partir de 1996 o AVE constitui causa principal de internações, mortalidade e deficiências na população brasileira ${ }^{13}$. Devido ao processo de envelhecimento da população, vem ocorrendo um aumento na expectativa de vida - e na incidência do AVE, com o avançar da ida$\mathrm{de}^{14,15}$, embora haja um declínio nas taxas de mortalidade no país ${ }^{14}$.

O AVE tem pico de incidência entre a sétima e oitava década de vida, quando se somam com as alterações cardiovasculares e metabólicas relacionadas à distribuídos quase igualmente por sexo. A média de idade dos pacientes foi $67,8 \pm 13,6$ anos; metade eram analfabetos; a maioria $(41,2 \%)$ tinha renda familiar de um salário mínimo. O tempo médio decorrido desde o AVE foi de $6,7 \pm 5,6$ anos

Em relação ao dimídio acometido, embora a distribuição seja semelhante entre o direito e o esquerdo (Tabela 1), $96 \%(n=49)$ dos participantes eram destros. Registrou-se ainda se que $35 \%$ $(n=18)$ dos pacientes nunca tinham tido tratamento fisioterapêutico e apenas $16 \%(n=8)$ o estavam recebendo na época da pesquisa. Quanto às faixas etárias, notou-se que as décadas de maior incidência do AVE foram as de 70-79 anos, 60-69 e 50-59 anos (Gráfico 1).

Em relação aos fatores de risco anteriores ao episódio de AVE, o tabagismo e o etilismo foram referidos por mais da

Tabela 2 Presença (\%) de fatores de risco anteriores ao primeiro episódio de acidente vascular encefálico informados pelos sujeitos

\begin{tabular}{|c|c|c|}
\hline Fatores & Sim & Não \\
\hline Dieta alimentar inadequada & 94 & 8 \\
\hline Inatividade física & 90 & 10 \\
\hline Tabagismo & 57 & 43 \\
\hline Etilismo & 55 & 45 \\
\hline Histórico de AVE na família & 33 & 66 \\
\hline
\end{tabular}

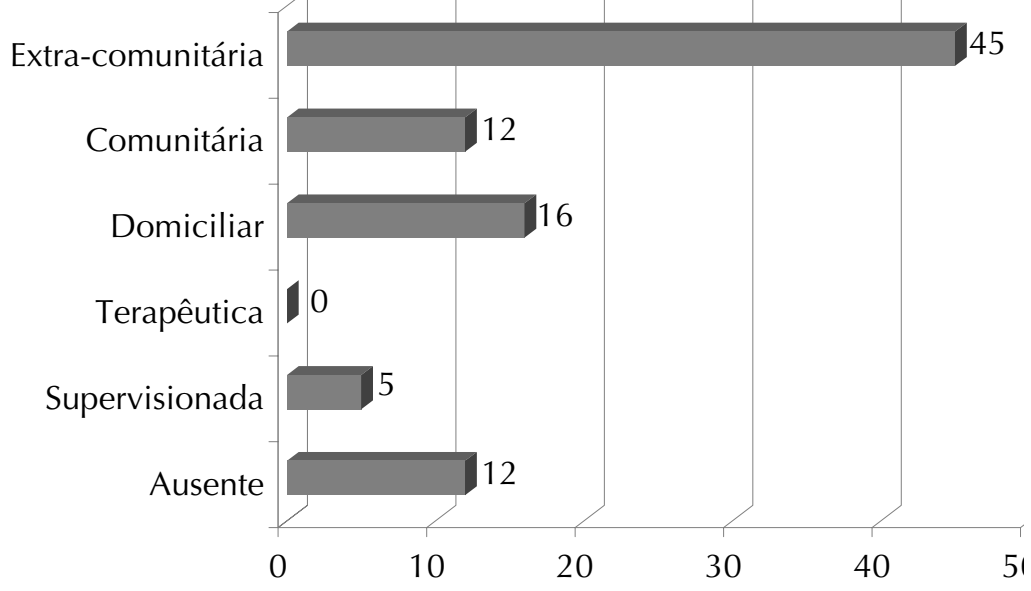

Gráfico 2 Distribuição (\%) dos tipos de marcha informados pelos sujeitos $(n=51)$

metade da amostra; a maioria (94\%) não realizava nenhum tipo de dieta alimentar prescrita por especialista e $90 \%$ não praticavam atividade física regularmente (Tabela 2). Também foi constatado que $78 \%(\mathrm{n}=40)$ dos indivíduos entrevistados eram hipertensos e 33\% $(n=17)$ tinham histórico de AVE na família.

No que se refere aos aspectos físicos, observou-se alteração na fala em 53\% $(n=27)$. Quanto à marcha, 78\% $(n=40)$ eram deambuladores, dos quais 23 apresentavam marcha extra-comunitária (Gráfico 2), sendo que 33\% (n=17) utilizavam algum dispositivo de auxílio à marcha. Poucos pacientes tiveram a marcha supervisionada (necessidade de auxílio eventual do cuidador ou do terapeuta) e nenhum apresentou marcha idade $^{16}$. Entretanto, pode ocorrer mais precocemente $^{16,17}$ e ser relacionado a outros fatores de risco, como distúrbios da coagulação, doenças inflamatórias e imunológicas, bem como o uso de dro$\operatorname{gas}^{16}$. Apesar do aumento da incidência, há que se considerar as taxas de sobrevivência que declinam com o avanço da idade - de $79 \%$ dos 75 aos 84 anos para $67 \%$ acima dos 85 anos $^{18}$-, o que poderia justificar a baixa incidência do AVE na faixa de 80 anos ou mais em nosso estudo.

O hemicorpo mais freqüentemente acometido em nossa população foi o esquerdo, contudo isso não parece ser relevante como fator prognóstico, pois a literatura mostra variações nessa freqüência ${ }^{19-21}$. 
Appelros et al..$^{22}$ realizaram uma revisão sistemática de 59 artigos sobre a incidência do AVE quanto ao sexo. Encontrou-se uma média de idade da incidência do primeiro episódio do AVE de 68,6 anos para homens, e 72,9 anos para mulheres. As taxas de incidência (33\%) e prevalência ( $41 \%$ ) foram maiores nos homens do que nas mulheres. Os valores referentes ao infarto encefálico e hemorragia intraencefálica foram maiores nos homens; as taxas referentes à hemorragia subaracnóidea foram maiores nas mulheres, embora essa diferença não seja estatisticamente significativa. Além do mais, o AVE tende a ser mais grave nas mulheres $(24,7 \%)$ quando comparado ao dos homens (19,7\%). No presente estudo, os sujeitos se distribuíram quase igualmente entre os sexos.

O aumento e o envelhecimento da população, somados aos fatores de risco mais prevalentes como hipertensão, tabagismo, dieta inadequada, inatividade física e obesidade, fazem com que o AVE se torne a principal causa de morte prematura e de incapacidade entre adultos $^{4}$. Assim, a detecção e o controle dos fatores de risco são tarefas prioritárias, pois permitem uma redução significativa da incidência e recidiva do $\mathrm{AVE}^{23,24}$ por meio de mudança nos hábitos de vida, terapêutica medicamentosa, neuro-radiologia intervencionista ou cirurgia $^{24}$.

Os fatores de risco para o AVE podem ser classificados em modificáveis, nãomodificáveis e ambientais. Os modificáveis são: hipertensão arterial sistêmica, tabagismo, inatividade física, dieta (baixa ingestão de frutas e vegetais), alcoolismo, obesidade e diabetes. Os não-modificáveis são: ocorrência prévia de ataque isquêmico transitório, hipercolesterolemia, estresse, cardiopatias, idade, sexo (por exemplo, alta idade e sexo masculino estão em muitas populações associados ao aumento do risco), raça (afrodescendentes), histórico familiar (fator genético); e fatores ambientais: fumantes passivos e acesso a tratamento médico ${ }^{1,3}$. Neste estudo, os fatores de risco modificáveis relatados foram dieta inadequada e inatividade física (pela quase totalidade dos participantes), tabagismo e alcoolismo (por mais de metade dos sujeitos - Tabela 2).
Em uma campanha inédita no Brasil, a Academia Brasileira de Neurologia uniu-se a um esforço mundial para realizar anualmente o "Dia Internacional do Acidente Vascular Cerebral" em várias capitais brasileiras, com o objetivo de conscientizar a população sobre a gravidade da doença, orientar para reconhecer os sinais e informar as providências necessárias diante de uma pessoa sofrendo de um AVE ${ }^{25}$. Salienta-se a importância da expansão desse programa de prevenção às demais localidades brasileiras.

Tem sido demonstrado que grupos populacionais economicamente desfavorecidos vêm apresentando taxas de mortalidade cardiovasculares mais elevadas do que os que possuem melhores condições de vida. Além de as camadas mais pobres da população também estarem submetidas aos principais fatores de risco, ainda usam produtos mais baratos, portanto de mais baixa qualidade. São consumidos cigarros que contêm alto teor de nicotina, bebidas alcoólicas destiladas (como cachaça), carnes com alto teor de gorduras, frituras com óleos saturados, enlatados etc. ${ }^{26}$.

Dados de 2006 da Pesquisa Nacional por Amostra de Domicílios, realizada pelo Instituto Brasileiro de Geografia e Estatística, apontam que 10,38\% da população se declaram analfabetos absolutos. O percentual representa 14,3 milhões de brasileiros ${ }^{27}$. Hoje vivemos na sociedade da informação e do conhecimento; quem não tem condições de ler e escrever acaba excluído de informações que são necessárias para garantir todos os seus direitos de cidadão, destacando-se o acesso à saúde.

Epidemiologicamente o AVE é uma doença altamente prevalente no Brasil. Dentre os sobreviventes, aproximadamente $70 \%$ recuperam sua marcha normal em um ano; 45 a 60\% são capazes de desempenhar seus cuidados pessoais com assistência; e 5 a 9\% tornam-se completamente independentes. Profissionalmente, 9\% retornam a seus trabaIhos originais, $1 \%$ mudam suas ocupações, $33 \%$ nunca retornam ao trabalho, e $57 \%$ permanecem desempregados ${ }^{5}$. Os pacientes apresentam alterações sensitivas, cognitivas e motoras como fraqueza muscular, espasticidade, padrões anormais de movimento e descondicio- namento físico. Esses défices podem limitar a capacidade de realizar tarefas funcionais como deambular, fazer compras, subir escadas e cuidar-se ${ }^{18}$. Falcão et al. ${ }^{17}$ encontraram défices de aproximadamente $80 \%$ entre os sobreviventes de AVE entrevistados, com seqüelas motoras em $75 \%$ entre os homens e $90 \%$ entre as mulheres. Além disso, pouco mais da metade das pessoas apresentavam impedimento para deslocar-se para outros bairros e entre as que se deslocavam havia necessidade da ajuda familiar e/ou do uso de dispositivos auxiliares, como bengala, cadeira de rodas, andador, entre outros. Similarmente, neste estudo, no que se refere à marcha, mais de $50 \%$ dos indivíduos apresentaram dificuldades de locomover-se de seus domicílios.

A hemiparesia não causa apenas limitações motoras, mas também interfere nas atividades de vida diária dos pacientes, com conseqüente redução da qualidade de vida. Fisioterapeutas têm demonstrado interesse em desenvolver e propor novas terapias de reabilitação e assim assegurar uma melhora de vida desses pacientes ${ }^{5}$. Segundo Duncan et al. ${ }^{28}$, uma reabilitação efetiva iniciada precocemente após o AVE pode aumentar os processos de recuperação e minimizar incapacidades funcionais, reduzindo os potenciais custos com cuidados a longo prazo e contribuindo com uma melhora da satisfação do paciente. Salienta-se que os pacientes crônicos com hemiplegia, quando submetidos à fisioterapia (treino de força muscular e condicionamento aeróbio), apresentam melhora da velocidade da marcha, maior capacidade de geração de força, aumento do $\mathrm{VO}_{2}$ máximo, melhora do desempenho funcional e da qualidade de vida $^{20,29}$.

A alta incidência de AVE na população atendida pelas ESF de Diamantina aponta para a necessidade de implantação e melhorias das políticas de saúde locais. Essas ações devem garantir um acompanhamento adequado desde a fase hospitalar até a domiciliar. Na fase domiciliar, salienta-se a importância da inserção do serviço de fisioterapia nas ESF, para identificar, avaliar e tratar os indivíduos com seqüelas neurológicas por AVE, atuar na prevenção dos fatores 
de risco e encaminhá-los aos centros de reabilitação especializados. São também necessários projetos de saúde que atinjam as camadas sociais mais carentes, como por exemplo a disponibilidade de veículos para garantir o acesso desses indivíduos a esses centros. Devido à alta incidência de AVE encontrado nas ESF, ações de saúde pública que diminuam os altos índices de morbidade e comorbidade dessa doença atuariam como indicadores de melhoria na saúde pública local. Porém, salienta-se que tais indicadores não devem ser analisados individualmente como parâmetros de base para todas as melhorias encontradas, uma vez que podem gerar um resultado falso-positivo. Novos estudos devem ser feitos nessa área, abrangendo não só os indivíduos com AVE, como também de outras doenças presentes no município, para que a Secretaria Municipal de Saúde possa implementar e/ou melhorar políticas de saúde adequadas a toda a população.

Com base nos resultados aqui obtidos, apresentados à Secretaria Municipal de Saúde de Diamantina, foram discutidas as políticas de ações na saúde, em especial das competências fisioterapêuticas para a população. Formou-se uma parceria entre a universidade e a Prefeitura Municipal para a viabilização de transporte dos pacientes que, sem condições financeiras, não têm acesso aos serviços ambulatoriais de fisioterapia e permanecem desamparados. Além disso, a inser- ção de profissionais fisioterapeutas nas ESF permitirá um novo cenário de atuação desses profissionais, para a abordagem integral do processo de saúde-doença, bem como para atuar na prevenção e orientação dos sujeitos, evitando novas doenças ou recidivas.

Segundo um parecer conjunto dos Conselhos Nacionais de Saúde e Educação ${ }^{30}$, é necessária a articulação entre saúde e ensino; em consonância, as diretrizes curriculares nacionais para o ensino superior na área da saúde contemplam a construção do perfil acadêmico e profissional com competências, habilidades e conteúdos contemporâneos, e para que os profissionais atuem com qualidade e resolubilidade no Sistema Único de Saúde.

O estudo realizado propõe uma ação conjunta entre município e universidade de modo que a integração ensinosaúde seja transformada em ações de políticas na saúde, gerando prestação de serviço gratuito e de qualidade à população. Além disso, a integração ensinosaúde possibilita a reflexão universitária e municipal, pois objetiva motivar e propor mudanças na formação técnica de graduação e pós-graduação, bem como viabilizar um processo de educação permanente dos trabalhadores da saúde, com base nas necessidades de saúde da população.

Apesar de não ter sido este o objetivo do estudo, é importante investigar direta- mente alguns parâmetros - o que poderá ser realizado em outros trabalhos como por exemplo medidas da pressão arterial, teste de equilíbrio e funcionalidade, além da aplicação de questionários validados que permitam avaliar depressão e qualidade de vida.

\section{CONCLUSÃO}

Observou-se neste estudo maior incidência do AVE com o avançar da idade, embora atingindo também indivíduos jovens. Além disso, revelou-se a presença, na população estudada, de fatores de risco como, dieta inadequada, inatividade física, tabagismo, etilismo, história de AVE paterno e/ou materno, baixa renda salarial e escolar. Embora tenham sido detectados défices funcionais relevantes na população dos sujeitos com hemiplegia, grande parte deles nunca receberam tratamento em fisioterapia. É nessa perspectiva que se percebe a importância da inserção da fisioterapia nas ESF, com o objetivo de atuar na prevenção e controle dos fatores de risco, bem como identificar, avaliar e tratar os indivíduos com seqüelas neurológicas do AVE, por meio de acompanhamento continuado. Ressalta-se ainda, a necessidade de melhorias ou modificações nas estratégias de políticas de saúde em relação à população estudada, bem como a importância da atuação conjunta entre município e universidade.

\section{REFERÊNCIAS}

1 World Health Organization (WHO). Steps stroke manual [periódico on-line] 2006 Mar-Apr [citado abr 2006]. Disponível em: http://www.who.int/en.

2 Thom T, Haase N, Rosamond W, Howard VJ, Faha JR, Faha TM, et al. Heart disease and stroke statistics: update; a report from the American Heart Association Statistics Committee and Stroke Statistics Subcommittee. Circulation. 2006;113:85-151.

3 O'Sullivan SB, Schimitz TJ. Fisioterapia: avaliação e tratamento. São Paulo: Manole; 2005.

4 Sridharan SE, MPhil U, Sukumaran S, Sylaja PN, Dinesh Nayak S, Sarma S, et al. Incidence, types, risk factors, and outcome of stroke in a developing country. Stroke. 2009;40:1112-8.
5 Fernandes MR, Carvalho LBC, Prado GF. A functional electric orthesis on the paretic leg improves quality of life of stroke patients. Arq Neuropsiquiatr. 2006;64(1)20-3.

6 Ellis C, Egede LE. Stroke recognition among individuals with stroke risk factors. Stroke. 2009;337(1)5-10.

7 Pontes-Neto OM, Silva GS, Feitosa MR, Figueiredo NL, Fiorot JA, Rocha TN, et al. Stroke awareness in Brazil. Stroke. 2008;39:292-6.

8 Mansur AP, Souza MFM, Favarato D, Avakian SD, César LAM, Aldrigui JM, et al. Stroke and ischemic heart disease mortality trends in Brazil from 1979 to 1996. Neuroepidemiology. 2003;22:179-83. 


\section{Referências (cont.)}

9 Brasil. Ministério da Saúde. Datasus. Mortalidade: Minas Gerais [citado set 2006]. Brasília; 2006. Disponível em: http://tabnet.datasus.gov.br/cgi/ deftohtm.exe?sim/cnv/obtMG.def.

10 Bueno DAA, Couto JMM, Leite HR, Nunes APN, Alves $\mathrm{PAB}$. Epidemiologia das doenças cerebrovasculares em Diamantina: 1998 a 2006 [monografia, CD-ROM]. Diamantina: Depto. de Ciências Básicas e da Saúde, Universidade Federal dos Vales do Jequitinhonha e Mucuri; 2008.

11 Folstein MF, Folstein SE, Mchugh PR. "Mini-mental state": a practical method for grading the cognitive state of patients for the clinicians. J Psychiatr Res. 1975;12:189-98.

12 Bertolucci PH, Brucki SM, Campacci SR, Juliano Y. O mini-exame do estado mental em uma população geral: impacto da escolaridade. Arq Neuropsiquiatr. 2004;52:1-7.

13 Brasil. Ministério da Saúde. Programas e projetos: doenças cardiovasculares. Brasília; 2000. Disponível em: http://www.saude.gov.br.

14 Minelli C, Fen LF, Minelli DPC. Stroke incidence, prognosis, 30-day, and 1-year case fatality rates in Matão, Brazil: a population-based prospective study. Stroke. 2007;38:2906-11.

15 Zarco LA, González F, Casas JC. Tratamiento actual del ataque cerebrovascular isquêmico (AVC) agudo. Univ Med Bogota (Colômbia) 2008;49(4):467-98.

16 Zétola VHF, Nóvak EM, Camargo CHF, Carraro Júnior $\mathrm{H}$ Coral P, Muzzio JA, et al. Acidente vascular cerebral em pacientes jovens: análise de 164 casos. Arq Neuropsiquiatr. 2001;59(3B)740-5.

17 Falcão IV, Carvalho EMF, Barreto KML, Lessa FJD, Leite VMM. Acidente vascular cerebral precoce: implicações para adultos em idade produtiva atendidos pelo Sistema Único de Saúde. Rev Bras Saude Matern Infant. 2004;4(1):95-102.

18 Teixeira-Salmela LF, Oliveira ESG, Santana EGS, Resende GP. Fortalecimento muscular e condicionamento físico em hemiplégicos. Acta Fisiatr. 2000;7(3)108-18.

19 Sommerfeld DK, Eek EUB, Svensson AK, Holmqvist LW, Arbin MHV. Spasticity after stroke: its occurrence and association with motor impairments and activity limitations. Stroke. 2004;35:134-40.
20 Macko RF, Ivey FM, Forrester LW, Hanley D, Sorkin J, Katzel LI, et al. Treadmill exercise rehabilitation improves ambulatory function and cardiovascular fitness in patients with chronic stroke: a randomized, controlled trial. Stroke. 2005;36;2206-11.

21 Vôos MC, Le RV. Estudo comparativo entre a relação do hemisfério acometido no acidente vascular encefálico e evolução funcional em indivíduos destros. Rev Bras Fisioter. 2008;12(2)113-20.

22 Appelros P, Stegmayr B, Terént. A. Sex differences in stroke epidemiology: a systematic review. Stroke. 2009;40:1082-90.

23 Araújo PS, Silva PCF, Moreira RCPS, Bonilha SF. Prevalência dos fatores de risco em pacientes com acidente vascular encefálico atendidos no Setor de Neurologia da Clínica de Fisioterapia da Unipar, campus sede. Arq Cienc Saude Unipar. 2008;12(1)35-42.

24 Pires SL, Gagliardi RJ, Gorzoni ML. Estudo das freqüências dos principais fatores de risco para acidente vascular cerebral isquêmico em idosos. Arq Neuropsiquiatr. 2004;62(3B):844-51.

25 Academia Brasileira de Neurologia (ABNeuro). Campanha Dia Nacional do Acidente Vascular Encefálico. ABNews. 2005;1:1-8.

26 Carvalho EMF, Branco MAF. Perfil de mortalidade por doenças cardiovasculares na região metropolitana de Recife, segundo o Sistema de Informação sobre Mortalidade (SIM). Informativo Epidemiol SUS. 1996;4:61-71.

27 Instituto Brasileiro de Geografia e Estatística (IBGE). PNAD 2006. Rio de Janeiro; 2007 [citado maio 2008]. Disponível em: http:// www.ibge.gov.br/home/estatistica/ populacao/ trabalhoerendimento/pnad2007/ default.shtm.

28 Duncan PW, Zorovitz R, Bates B, Choi JY, Glasberg JJ, Graham GD, et al. Management of adult stroke rehabilitation care: a clinical practice guideline. Stroke. 2005;36;e100-43.

29 Potempa K, Lopez M, Braun LT, Szidon P, Fogg L, Tincknell T. Physiological outcomes of aerobic exercise training in hemiparetic stroke patients. Stroke. 19995;26:101-5.

30 Brasil. Conselho Nacional de Educação, Conselho Nacional de Saúde. Parecer CNE/CES 1.300/2001; despacho do Ministro em 4/12/2001. DOU. 7 dez 2001; seção 1:25. 